\title{
flan: An R Package for Inference on Mutation Models.
}

\author{
by Adrien Mazoyer, Rémy Drouilhet, Stéphane Despréaux, and Bernard Ycart
}

\begin{abstract}
This paper describes flan, a package providing tools for fluctuation analysis of mutant cell counts. It includes functions dedicated to the distribution of final numbers of mutant cells. Parametric estimation and hypothesis testing are also implemented, enabling inference on different sorts of data with several possible methods. An overview of the subject is proposed. The general form of mutation models is described, including the classical models as particular cases. Estimating from a model, when the data have been generated by another, induces different possible biases, which are identified and discussed. The three estimation methods available in the package are described, and their mean squared errors are compared. Finally, implementation is discussed, and a few examples of usage on real data sets are given.
\end{abstract}

\section{Introduction}

Mutation models are probabilistic descriptions of the growth of a population of cells, where mutations occur randomly during the process. Data are samples of integers, interpreted as final numbers of mutant cells. These numbers may be coupled with final numbers of cells (mutant and non mutant). The frequent appearance in the data of very large mutant counts, usually called "jackpots", evidences heavy-tailed probability distributions. The parameter of interest is the mutation probability for a mutant cell to appear upon any given cell division, denoted by $\pi$. In practice, $\pi$ is typically of order $10^{-9}-10^{-11}$. Computing robust estimates for $\pi$ is of crucial importance in medical applications, like cancer tumor relapse or multidrug resistance of Mycobacterium Tuberculosis for instance.

Any mutation model can be interpreted as the result of the three following ingredients:

- a random number of mutations occurring with small probability among a large number of cell divisions. Due to the law of small numbers, the number of mutations approximately follows a Poisson distribution. The expectation of that distribution, denoted by $\alpha$, is the product of the mutation probability $\pi$ with the total number of divisions;

- from each mutation, a clone of mutant cells growing for a random time. Due to exponential growth, most mutations occur close to the end of the experiment, and the developing time of a random clone has exponential distribution. The rate of that distribution, denoted by $\rho$, is the relative fitness, i.e. the ratio of the growth rate of normal cells to that of mutants;

- the number of mutant cells that any clone developing for a given time will produce. The distribution of this number depends on the distribution of division times of mutants.

Using the theory of continuous time branching processes (Bellman and Harris, 1952; Athreya and Ney, 1972), and under specific modeling assumptions, it can be proved that the asymptotic distribution of the final number of mutants has an explicit form. A first mutation model with explicit distribution is the well known Luria-Delbrück model (Luria and Delbrück, 1943). Other mathematical models were introduced by Lea and Coulson (1949), followed by Armitage (1952) and Bartlett (1978). In these models, division times of mutant cells were supposed to be exponentially distributed. Thus a clone develops according to a Yule process, and its size at a given time follows a geometric distribution. The distribution of final mutant counts is also explicit when division times are supposed to be constant. This latter model is called Haldane model by Sarkar (1991); an explicit form of the asymptotic distribution is given in Ycart (2013). General division times have been studied by Ycart (2013), but no explicit distribution is available apart from the exponential and constant division times.

The first estimation method was given by Luria and Delbrück (1943). It is based on the simple relation between the probability of null counts in the sample, and the mutation probability, and it is called P0 method. Of course, if the sample does not contain null counts, the method cannot be applied. Apart from the P0 method, all other methods couple the estimation of $\pi$ or $\alpha$, with the estimation of $\rho$. When the distribution of final numbers has an explicit form, the Maximum Likelihood (ML) is an obvious optimal choice (Ma et al., 1992; Zheng, 2005). However, because of the jackpots, likelihood computation can be numerically unstable. There are several ways to reduce tail effects (Wilcox, 2012, Sec. 2.2), among which "Winsorization" consists in truncating the sample beyond some maximal value. Another estimation method (GF) uses the probability generating function (PGF) (Rémillard and Theodorescu, 2000; Hamon and Ycart, 2012). The estimators of $\alpha$ and $\rho$ obtained with the GF method proved to be close to optimal efficiency, with a broad range of calculability, a good numerical stability, and a negligible computing time. For the three methods, P0, ML, and GF, the estimators of $\alpha$ and 
$\rho$ are asymptotically normal. Thus confidence intervals and $\mathrm{p}$-values for hypothesis testing can be computed, for one sample and two sample tests.

The problem with classical mutation models, is that they are based on quite unrealistic assumptions: constant final number of cells (Angerer, 2001a; Komarova et al., 2007; Ycart and Veziris, 2014), no cell deaths (Angerer (2001a, Sec. 3.1); Dewanji et al. (2005); Komarova et al. (2007); Ycart (2014)), fully efficient plating (Stewart et al., 1990; Stewart, 1991; Angerer, 2001b), or, as mentioned above, exponential distribution of division times. Using a model for estimation, when the data have been generated by another one, necessarily induces a bias on estimates. For instance, if cell deaths are neglected, mutation probability will be underestimated.

Several informatic tools have already been developed for fluctuation analysis (Zheng, 2002; Hall et al., 2009; Gillet-Markowska et al., 2015). These tools are quite user-friendly. However, they do not take into account all the possible model assumptions mentioned above. The flan package described here, is dedicated to mutation models, and parameter estimation with the three methods P0, ML, and GF. It includes a set of functions for the distribution of mutant cell counts (dflan, pflan, qflan, $r f l a n$ ) and a graphic function (draw. clone). They treat general models, with fluctuating final numbers, cell deaths, and other division time distributions than exponential and constant. The general estimation function is mutestim. It returns estimates for the parameters $\alpha, \pi$ and $\rho$, with the three estimation methods, constant or exponential division times, and cell deaths. As a wrapper, a hypothesis testing function (flan. test) is provided. In order to make the package user-friendly, the functions have been designed to resemble classical $R$ functions, like t. test or rnorm.

The paper is organized as follows. Section 2.2 is devoted to the probabilistic setting: the hypotheses of the different models are described, and the asymptotic results are explained. In Section 2.3, the three estimation methods are exposed, and the biases described above are discussed. A comparison of the three methods in terms of mean squared errors is provided. The user interface and the Rcpp (Eddelbuettel, 2013) implementation is treated in Section 2.4; examples of execution are shown in Section 2.5.

\section{Mutation models}

In this section, probabilistic mutation models are described. The basic modeling hypotheses are the following:

- at time 0 a homogeneous culture of $n_{0}$ normal cells is given;

- the lifetime of any normal cell is a random variable with distribution function $F$;

- upon completion of the generation time of a normal cell:

- with probability $\pi$ one normal and one mutant cell are produced;

- with probability $1-\pi$ two normal cells are produced;

- the lifetime of any mutant cell is a random variable with distribution function $G$;

- upon completion of the lifetime of a mutant cell:

- with probability $\delta$ the cell dies out;

- with probability $1-\delta$ two mutant cells are produced;

- all random variables and events (division times, mutations, and deaths) are mutually independent.

Consider that the initial number $n_{0}$ tends to infinity, the mutation probability $\pi=\pi_{n_{0}}$ tends to 0 , and the time $t=t_{n_{0}}$ at which mutants are counted tends to infinity. The scale of time is supposed to be adjusted so that the exponential growth rate of mutants is 1 ; thus the exponential growth rate of normal cells is $\rho$. See Athreya and Ney (1972, Chap. IV Sec. 4) or Hamon and Ycart (2012) for the definition of the growth rate (also called "Malthusian parameter"). The expected number of mutations before $t_{n_{0}}$ is proportional to $n_{0} \pi_{n_{0}} \mathrm{e}^{\rho t_{n_{0}}}$, and the asymptotics are assumed to be such that this number converges as $n_{0}$ tends to infinity to $\alpha$, positive and finite.

Under the above hypotheses, as $n_{0}$ tends to $+\infty$, the final number of mutants converges in law to the distribution with PGF:

with

$$
g(z)=\exp (-\alpha(1-h(z)))
$$

$$
h(z)=\int_{0}^{\infty} \psi(z, t) \rho \mathrm{e}^{-\rho t} \mathrm{~d} t,
$$

where $\psi(z, t)$ is the PGF of the number of cells at time $t$ in a mutant clone, starting from a single cell at time 0 . Observe that it depends on the lifetime distribution of normal cells $F$ only through $\rho$. The 
above result is deduced from the theory of continous time branching processes (Hamon and Ycart, 2012)). The expressions (1) and (2) translate the three ingredients described in the introduction:

1. the Poisson distribution with intensity $\alpha$ models the total number of mutations which occur during the process;

2. the exponential distribution with rate $\rho$ is that of the time during which a random clone develops;

3. the distribution with PGF $\psi(\cdot, t)$ is that of the number of cells in a random clone developing during a time interval of length $t$. The PGF $\psi$ is the solution of a Bellman-Harris equation (Bellman and Harris, 1952) in terms of $\delta$ and $G$.

Hence the expressions of $h$ as an exponential mixture, and of $g$ as a Poisson compound. In practice, the plating process can be less than $100 \%$ efficient. In that case, a random number of mutants will not be counted: if only a proportion $\zeta$ of the final population is plated, then each cell will be observed with probability $\zeta$. Denote by $M_{\text {tot }}$ and $M$ the total and the observed numbers of mutants. Given $M_{\text {tot }}=m$, $M$ follows the binomial distribution with parameters $m$ and $\zeta$. Thus, the PGF $g$ of $M$ is given by:

$$
\begin{aligned}
g(z) & =\mathbb{E}\left[\mathbb{E}\left[z^{M} \mid M_{\text {tot }}\right]\right] \\
& =\exp (-\alpha(1-h(1-\zeta+\zeta z))) .
\end{aligned}
$$

The PGF (3) defines a parametrized family of distributions, denoted hereafter by $M M(\alpha, \rho, \delta, \zeta, G)$ (Mutation Model). This is a family of heavy-tailed distributions, with tail exponent $\rho$ : the higher the fitness, the heavier the tail. This directly influences the number and the amount of jackpots.

At this point, the PGF $\psi$ can be given as an explicit expression only for two particular lifetime distributions of mutants: exponential, and Dirac (constant lifetimes). The corresponding mutation models will be denoted respectively by $\operatorname{LD}(\alpha, \rho, \delta, \zeta)$ (Luria-Delbrück), and $H(\alpha, \rho, \delta, \zeta)$ (Haldane). The functions dflan, pflan, and qflan compute densities, probabilities, quantiles of $L D$ and $H$ distributions (with $\zeta=1$ ).

Assuming that a consistent estimator of $\alpha$ has been defined, the problem in practice is to compute reliable estimates of the mutation probability, $\pi$. The simplest approach assumes that the final number of cells, denoted by $N$, is constant. An estimate of $\pi$ is then obtained by dividing the estimate of $\alpha$ by $N$. However, even under close experimental monitoring, assuming that the final number of cells is a constant is quite unrealistic. Thus, $N$ must be viewed as a random variable with a certain probability distribution function $K$ on $[0,+\infty)$. By analogy with (1), the conditional PGF of the number of mutants given $N=n$, can be given by the following expression:

$$
g(z \mid N=n)=\exp (-\pi n(1-h(z))) .
$$

Or else, the conditional distribution of the number of mutants given $N=n$ is the distribution $M M(\pi n, \rho, \delta, \zeta, G)$. The distribution function $K$ is supposed to be known and its Laplace transform is denoted by $\mathcal{L}$ :

$$
\mathcal{L}(z)=\mathbb{E}\left[\mathrm{e}^{-z N}\right]=\int_{0}^{\infty} \mathrm{e}^{-z n} \mathrm{~d} K(n),
$$

Thus the PGF of the final number of mutants is given by:

$$
\begin{aligned}
g(z) & =\int_{0}^{\infty} g(z \mid N=n) \mathrm{d} K(n) \\
& =\mathcal{L}(\pi(1-h(z))) .
\end{aligned}
$$

Remark that if $N$ is constant, (4) reduces to (1) with $\alpha=\pi N$. In general, the PGF (4) defines a new parametrized family of mutation distributions, denoted herafter by $\operatorname{MMFN}(\pi, \rho, \delta, \zeta, G, K)$ (Mutation Models with Fluctuating Numbers of cells).

The two particular cases for the distribution GX previously mentioned above (exponential and Dirac) will be denoted by $\operatorname{LDFN}(\alpha, \rho, \delta, \zeta, K)$ (Luria-Delbrück with Fluctuating Number of cells) and $\operatorname{HFN}(\alpha, \rho, \delta, \zeta, K)$ (Haldane with Fluctuating Number of cells). As will be shown in Section 2.3, estimating $\pi$ by the ratio of an estimate of $\alpha$ by the expectation of $N$ induces a negative bias.

The function $\mathrm{rflan}$ outputs samples of pairs (mutant counts-final counts) following MMFN distributions where $G$ is an exponential, Dirac, log-normal or gamma distribution, and $K$ is a lognormal or Dirac distribution. 


\section{Statistical inference}

Here the three estimation methods $\mathrm{P} 0, \mathrm{ML}$ and GF are described. The main features and the limitations of each method are discussed. The three methods compute estimates of $\alpha$ and $\rho$, under the $L D$ and $H$ models. When couples (mutant counts-final numbers) are given, estimates of $\pi$ and $\rho$ are calculated under the LDFN or HFN models.

Even if the probabilities and their derivatives with respect to $\delta$ for $L D$ and $H$ distributions can be computed, the variations of the whole distribution as a function of $\delta$ are too small to enable estimation in practice (see Ycart (2014) for more details). Thus, the parameter $\delta$ is supposed to be known for the three methods.

In the rest of this section, the three estimators are described, their performances compared in terms of MSE, and the possible sources of biases discussed.

\section{Estimators}

P0 estimator: The first method was introduced by Luria and Delbrück (1943) when $\delta=0$. In that case, the probability of null counts in the sample is $\mathrm{e}^{-\alpha}$. Hence $\alpha$ can be estimated taking the negative logarithm of the relative frequency of zeros among mutant counts. Hence the method cannot be applied if the sample does not contain null counts.

If $\delta>0$, the probability of null counts in the sample depends also on $\delta$. Assuming $\delta<1 / 2$, a fixed point of the PGF $\psi(\cdot, t)$ is the extinction probability of a mutant clone (Athreya and Ney, 1972, Theorem 1, Chap.I):

$$
\delta_{*}=\frac{\delta}{1-\delta} .
$$

By definition, $\delta_{*}$ is also a fixed point of the PGF (2). Then the probability of null counts in the sample is $\mathrm{e}^{-\alpha\left(1-\delta_{*}\right)}$. A consistent and asymptotically normal estimator of $\alpha$ is given by:

$$
\hat{\alpha}_{0}=\frac{-\log \left(\hat{g}\left(\delta_{*}\right)\right)}{1-\delta_{*}},
$$

where $\hat{g}$ denotes the empirical PGF of the final number of mutants.

Consider now that $\zeta<1$. Ignoring the inefficient plating will induce a negative bias. A correction has been proposed by Stewart et al. (1990, eq. (41)). However, it can be used only under


which depends on the fitness $\rho$. It is still possible to extend the estimator (5) to the case where $\zeta<1$ :

$$
\hat{\alpha}_{0}=\frac{-\log \left(\hat{g}\left(\delta_{*}^{(\zeta)}\right)\right)}{1-\delta_{*}},
$$

with

$$
\delta_{*}^{(\zeta)}=\frac{\delta_{*}-(1-\zeta)}{\zeta}
$$

We remark that (6) makes sense only if $\left|\delta_{*}^{(\zeta)}\right| \leqslant 1$. In particular, if $\delta=0$, the plating efficiency $\zeta$ has to be greater than 0.5 . Therefore, the

Notice that the P0 method does not directly yield an estimator of $\rho$. If an estimate is desired, the ML method can be used for $\rho$ only, setting $\alpha=\hat{\alpha}_{0}$.

ML estimators: Since algorithms (Embrechts and Hawkes, 1982; Zheng, 2005; Hamon and Ycart, 2012; Ycart and Veziris, 2014) enable the computation of the probabilities of the $L D$ and $H$ models, the ML method seems to be an obvious choice. It can be used on two kinds of samples:

1. sample of mutant counts: In that case, the likelihood is computed with the probabilities of the model $L D$ or $H$. The parameter of interest is $\alpha$.

2. sample of pairs of (mutant counts-final numbers): In that case, the likelihood is computed with the probabilities of the model LDFN or HFN. The parameter of interest is $\pi$.

In both cases, $\rho$ can also be estimated.

However, when the sample maximum is large, sums of products of small terms must be computed (Hamon and Ycart, 2012). The procedure can be very long and numerically unstable. Thus, the ML estimators can fail for large $\alpha$ and small $\rho$. In practice, this instability problem is avoided using Winsorization (Wilcox, 2012, Sec. 2.2), which consists in replacing any value of the sample that exceeds a certain bound by the bound itself. The bound is 1024 by default, and it could be necessary to increase 
it. All information above the bound is lost, and in an extreme case where the sample minimum is greater than the bound, irrelevant results will be returned.

In theory, it is also possible to be explicit about the probabilities of the $L D$ model when $\zeta<1$. However, these computations have not been done for the $H$ model. Thus the plating process is assumed to be fully efficient when the ML method is used.

GF estimators: The GF method uses the PGF to estimate the parameter of a compound Poisson distribution (Rémillard and Theodorescu, 2000; Hamon and Ycart, 2012). Let $0<z_{1}<z_{2}<1$ and $z_{3}$ in $(0 ; 1)$. The estimators of $\alpha$ and $\rho$ are the following:

$$
\hat{\alpha}_{G F}\left(z_{3}\right)=\frac{\log \left(\hat{g}\left(z_{3}\right)\right)}{h_{\hat{\rho}_{G F}\left(z_{1}, z_{2}\right)}\left(z_{3}\right)-1} \quad \text { and } \quad \hat{\rho}_{G F}\left(z_{1}, z_{2}\right)=f_{z_{1}, z_{2}}^{-1}(\hat{y}),
$$

where $\hat{g}$ denotes the empirical PGF of the final number of mutants, $h_{x}$ is the PGF (2) with $\rho=x$, and:

$$
f_{z_{1}, z_{2}}(x)=\frac{h_{x}\left(z_{1}\right)-1}{h_{x}\left(z_{2}\right)-1} \quad \text { and } \quad \hat{y}=\frac{\log \left(\hat{g}\left(z_{1}\right)\right)}{\log \left(\hat{g}\left(z_{2}\right)\right)} .
$$

From Rémillard and Theodorescu (2000), it can be proved that the couple of estimators $\left(\hat{\alpha}_{G F}, \hat{\rho}_{G F}\right)$ is strongly consistent and asymptotically normal, with explicit asymptotic variance (Hamon and Ycart, 2012).

The GF estimators depend on the three arbitrary values of $z_{1}, z_{2}, z_{3}$. Those tuning parameters are set to $z_{1}=0.1, z_{2}=0.9$, and $z_{3}=0.8$. For more details about the choice of those values, see Hamon and Ycart (2012).

In practice, the GF estimators are quite comparable in precision to ML estimators, with a much broader range of calculability, a better numerical stability, and a negligible computing time, even in the case where the ML method fails. For that reason, we have chosen to initialize the ML optimization by GF estimates, to improve both numerical stability and computing time.

The only practical limitation of this method is the following. A zero of the monotone function $f_{z_{1}, z_{2}}(\rho)-\hat{y}$ must be computed. An upper bound for the domain of research must be given, which can be a problem if the sample does not contain jackpots. However in that case, a mutation model is not adapted.

According to (3), the GF estimators or $\alpha$ and $\rho$ can be extended to the case where $\zeta \leqslant 1$ as follows:

$$
\hat{\alpha}_{G F}\left(z_{3}\right)=\frac{\log \left(\hat{g}\left(z_{3}\right)\right)}{h_{\hat{\rho}_{G F}\left(z_{1}, z_{2}\right)}\left(1-\zeta+\zeta z_{3}\right)-1} \quad \text { and } \quad \hat{\rho}_{G F}\left(z_{1}, z_{2}\right)=f_{1-\zeta+\zeta z_{1}, 1-\zeta+\zeta z_{2}}^{-1}(\hat{y})
$$

where $f_{z_{1}, z_{2}}$ and $\hat{y}$ are still given by (7). By the same reasoning as Hamon and Ycart (2012), strong consistency and asymptotic normality of the couple $\left(\hat{\alpha}_{G F}, \hat{\rho}_{G F}\right)$ can be proved.

The function mutestim computes estimates and their respective standard deviations for $\alpha, \pi$ and $\rho$ according to the type of input. Moreover, the estimators mentioned here are asymptotically normal. Thus, one and two sample tests can be performed, using the function flan. test. The null hypothesis will be either fixed theoretical values of $\alpha, \pi, \rho$ in the one sample case, or a difference of the same in the two sample case.

\section{Comparison of the three estimators}

The Figures 1 and 2 (drawn using ggplot2) show "maps of usage" of the estimation methods under the $L D$ and $H$ models. The methods are compared in terms of the relative $\operatorname{MSE}$ of $(\hat{\alpha}, \hat{\rho})$ defined as:

$$
\sqrt{\left(1-\frac{\hat{\alpha}}{\alpha}\right)^{2}+\left(1-\frac{\hat{\rho}}{\rho}\right)^{2}}
$$

The RGB code is used: red for GF, green for P0, blue for ML. Twenty values of $\alpha$ between 0.5 and 10 , and as many values of $\rho$ from 0.2 to 5 , were chosen. Thus 400 couples were considered. For each of them, the following procedure was applied:

1. draw $10^{4}$ samples of size 100 of the $\operatorname{LD}(\alpha, \rho, 0,1)$;

2. for each sample, compute ML, GF and P0 estimates of $(\alpha, \rho)$ under $L D$ model;

3. from the $10^{4}$ estimates, compute the relative MSEs of each method;

4. assign a RGB color according to the MSEs. For each method:

- if the MSE is less than 0.05, assign 1 to the corresponding RGB component; 




Figure 1: Map of usage of the estimation methods under $L D$ model. The map compares the three methods according to their relative MSE (8). For each of 400 couples of parameters $\alpha=0.5 \ldots 10$ (x-axis) and $\rho=0.2 \ldots 5$ (y-axis, $\log _{5}$-scale), $10^{4}$ samples of size 100 of the $L D(\alpha, \rho, 0,1)$ distribution were simulated. The estimates of $(\alpha, \rho)$ were calculated with the three methods. Each method is represented by a color: red for GF, green for P0, blue for ML.

- if the MSE is greater than 1, assign 0 to the corresponding RGB component;

- else, assign 1 minus the MSE to the corresponding RGB component.

The above experience is also performed considering $H$ model instead of $L D$. The maps have been drawn with a $\log _{5}$-scale for $\rho$ (y-axis). The map can be roughly divided into four distinct parts:

- For $(\alpha, \rho) \in(0.5 ; 3) \times(0.2 ; 2.5)$, the color is essentially grey: the three methods are more or less equivalent.

- For $(\alpha, \rho) \in(3 ; 10) \times(0.2 ; 3.5)$, the color is magenta: the ML and GF methods are equivalent. The P0 method provides estimates with large MSEs or cannot be used because of the absence of null counts.

- For small values of $\rho$, the color is mainly red: The GF method is the only method with an acceptable MSE. Small values of $\rho$ induce large jackpots. Moreover, the number of jackpots increases with $\alpha$. Because of the Winsorization, the ML and P0 method (which uses ML to estimate $\rho$ ) provide estimates with very large MSEs.

- For $\rho$ large, the color is darker and tends to black: the three methods provide estimates with large MSEs, specially for $\rho \in(3.5 ; 5)$, where jackpots are very small or absent. In those cases, estimating $\rho$ with the GF method is not possible in practice (see previous sub-section). Consequently, the GF method will provide a biased estimate for $\alpha$. The ML method, which uses the GF estimates to initialize the optimization of the log-likelihood, also provides biased estimates. The P0 method can provide good estimates of $\alpha$ whatever the value of $\rho$, which explains the presence of green areas at the top of the map. In a case where no jackpots are present in the sample it should be considered that a (heavy tailed) mutation model is not adapted.

Figure 2 is quite similar to Figure 1. There are still two remarkable differences:

- For $\rho$ small and $m \leqslant 2$, the three methods seem to be more equivalent under $H$ model than under $L D$ model.

- For $\rho$ large, GF method seems to provide better estimates under $H$ model than under $L D$ model.

The three methods should also be compared in terms of computational time. An illustration on real data will be given in section 2.5. The slowest method is ML, for the reasons discussed in the previous section. It is even slower when the estimates are calculated under Haldane models $H$ or $H F N$, when $\delta$ is positive, or if the initialization of $\rho$ with the GF method fails. The GF method computes estimates of $\alpha$ and $\rho$ (when possible) in negligible time. The P0 method outputs estimates of $\alpha$ in negligible time, but estimates of $\rho$ are as slow as with ML. 




Figure 2: Map of usage of the estimation methods under $\boldsymbol{H}$ model. The map compares the three methods according to their relative MSE (8). For each of 400 couples of parameters $\alpha=0.5 \ldots 10$ (x-axis) and $\rho=0.2 \ldots 5$ (y-axis, $\log _{5}$-scale), $10^{4}$ samples of size 100 of the $H(\alpha, \rho, 0,1)$ distribution were simulated. The estimates of $(\alpha, \rho)$ were calculated with the three methods. Each method is represented by a color: red for GF, green for P0, blue for ML.

\section{Bias evidence}

If the model used for the estimation does not correspond to the theoretical model, the estimates can be biased. Four different sources of bias are considered:

1. the final counts are random in the data, constant for the estimation model;

2. cell deaths occur in the data, not in the estimation model;

3. the lifetime distribution is different in the data and the estimation model;

4. the plating process is less than $100 \%$ efficient.

In each case, simulation experiments have been made along the following lines:

1. draw $10^{4}$ samples of size 100 , under one model;

2. for each sample, compute estimates using another model and the true one if available;

3. compare the empirical distributions of $\hat{\theta} / \theta$, where $\hat{\theta}$ is estimator and $\theta$ the true value.

For each figure, red lines mark unit, blue lines mark relative biases of 0.9 and 1.1. According to Figures 1 and 2, the GF method is at least equivalent in terms of the relative MSE (8) to the ML and P0 methods. Moreover, it is also the best in terms of computational time. Therefore, the bias evidences will be mainly illustrated with GF method.

Fluctuation of final counts: When $N$ is constant, the estimate of $\pi$ is derived by dividing the estimate of $\alpha$ by $N$. As mentioned in previous section, if $N$ is a random variable, the relation between $\alpha$ and $\pi$ can be explicit if the distribution $K$ is known. However, this is not the case in practice. Usually, estimates of the expectation and variance of $N$ are available at best. Assume that only the first two moments $\mu$ and $\sigma^{2}$ of $N$ are known. Then a first order approximation of the Laplace transform $\mathcal{L}$ can be used to reduce the bias. This method is explained in Ycart and Veziris (2014) for the P0 method. It has been adapted to ML and GF estimates. Figure 3 shows the influence of the coefficient of variation $C=\sigma / \mu$ on the ML estimate of $\pi$. The estimates were calculated with three different approaches:

- divide ML estimates of $\alpha$ by the empirical mean of $N$ and ignore fluctuations of $N$ (left boxplots);

- directly compute ML with the sample of pairs (mutant counts-final counts) (center boxplots);

- derive from ML estimates of $\alpha$, taking into account of the empirical fluctuations of $N$ (right boxplots). 
$\pi=5 \times 10^{-10}$


$\pi=2 \times 10^{-9}$


$\pi=4 \times 10^{-9}$

$\pi=8 \times 10^{-9}$


Figure 3: ML estimates of $\pi$ ignoring fluctuations of final numbers or not. Red horizontal lines mark unit. Blue horizontal lines mark relative bias of 0.9 and 1.1. For each of the 12 sets of parameters $\pi=(0.5 / \mu, 2 / \mu, 4 / \mu, 8 / \mu)$ (columns), and $C=(0.2,0.4,0.6)$ (rows), $10^{4}$ samples of size 100 of the $\operatorname{LDFN}(\pi, \rho, 0,1, K)$ distribution were simulated, with $\rho=1$ and $K$ being the log-normal distribution adjusted to mean $\mu=10^{9}$ and coefficient of variation $C$. The ML estimates of $\pi$ and $\rho$ were calculated under model $L D$ or $L D F N$. Each boxplot represents the distribution of the $10^{4}$ ratio $\hat{\pi} / \pi$ obtained with $L D$ model with $C=0$ (left), $L D$ model with bias reduction (center), $L D F N$ model (right).

According to the visual observations, the bias reduction seems to be working well when either the product $\pi \mu$ or $C$ are small: most of the estimates have a relative bias smaller than $10 \%$. However, the efficiency of the correction decreases as product $\pi \mu$ and $C$ increase. In particular, for larger values of $\pi \mu$, the bias seems to be smaller without correction. It could be improved with a better approximation of $\mathcal{L}$, that implies knowing or estimating higher moments of $N$. Another solution is to improve the estimation of $C$. Here $C$ was estimated by the ratio of the empirical standard deviation of the empirical mean, which is known to be a bad method in terms of MSE (Breunig, 2001).

Cell deaths: The PGFs (1), (4) and (2) depend on $\delta$. Ignoring cell deaths involves a negative bias on the estimate of $\alpha$. Assuming the exact value is known, this bias is removed. Figure 4 shows the influence of the death parameter $\delta$ on the GF estimate of $\alpha$. The estimates are calculated with two different approaches:

1. computing GF estimates of $\alpha$ with $\delta=0$ (left boxplots);

2. computing GF estimates of $\alpha$ with a theoretical value of $\delta$ (right boxplots).

The visual results show that the negative bias induced by ignoring cell deaths increases with the value of $\delta$ : the relative bias can easily exceed $10 \%$ for large values of $\delta$. From the theory of branching processes, the growth process of a mutant clone is supercritical and $\delta$ has to be smaller than 0.5 . In practice $\delta$ is smaller than 0.3 . According to the boxplots, the relative bias induced by ignoring cell deaths can reach 0.80 . These experiments also illustrate the difficulty in estimating $\delta$. For example, the boxplots at the top right of the figure seems to show that the value of the likelihood for $\alpha=4$ and $\delta=0$ is very close to its value for $\alpha=4$ and $\delta=0.05$. 
$\alpha=0.5$

$\delta=0.05$


$\delta=0.2$



$\alpha=2$


$\alpha=4$


$\alpha=8$


Figure 4: GF estimates of $\alpha$ ignoring cell deaths or not. Red horizontal lines mark unit. Blue horizontal lines mark relative bias of 0.9 and 1.1. For each of the 12 sets of parameters $\alpha=(0.5,2,4,8)$ (columns), and $\delta=(0.05,0.1,0.2)$ (rows), $10^{4}$ samples of size 100 of the $L D(\alpha, \rho, \delta, 1)$ distribution were simulated, with $\rho=1$. The GF estimates of $\alpha$ and $\rho$ were calculated under $L D$ model. Each boxplot represents the distribution of the $10^{4}$ ratio $\hat{\alpha} / \alpha$ of the estimates obtained without taking account of cells death (left) and with the theoretical value of $\delta$ (right).

Lifetime distribution: As mentioned earlier, the PGF $h$ is explicit only for the $L D$ and $H$ distributions, i.e. when lifetimes are either exponential or constant. This is not the case in practice. If another lifetime distribution is used to simulate the data, and either $L D$ or $H$ are used to estimate the parameters, a bias will be induced on $\alpha$ and $\rho$. Figure 5 illustrates these observations. It shows the influence of the lifetime distribution on the GF estimates of $\alpha$ and $\rho$. The samples are drawn assuming the lifetimes are log-normally distributed. The estimates of $\alpha$ and $\rho$ are calculated under $L D$ (left boxplots) and $H$ models (right boxplots).

From the visual observations, the $L D$ and $H$ models can be seen as extreme values for the lifetime distribution:

- both models correctly estimate $\alpha$;

- the $L D$ model overestimates $\rho$ and has a rather large dispersion of estimated values. The bias seems to increase as $\alpha$ increases;

- the $H$ model correctly estimates $\rho$.

Plating efficiency: Ignoring the plating efficiency induces a negative bias on $\alpha$ and a positive bias on $\rho$. In practice, the exact value is known. Figure 6 shows the influence of $\zeta$ on the GF estimates of $\alpha$. The estimates are calculated with two different approaches:

1. computing GF estimates with $\zeta=1$ (left boxplots) ;

2. applying the correction of Stewart et al. (1990, eq. (41)) to GF estimates (center boxplots);

3. computing GF estimates with known value of $\zeta$ (right boxplots).

The visual results illustrate first the fact that the correction of Stewart et al. (1990) should not be used when $\rho \neq 1$ : the induced bias on $\alpha$ is negative when $\rho>1$, positive when $\rho<1$. However, the variance 




Figure 5: GF estimates of $\alpha$ and $\rho$ under $L D$ and $H$ models on data drawn with $M M$ model. Red horizontal lines mark unit. Blue horizontal lines mark relative bias of 0.9 and 1.1. For each of the 12 sets of parameters $\alpha=(0.5,2,4,8)$ (rows), and $\rho=(0.8,1,1.2)$ (columns), $10^{4}$ samples of size 100 of the $M M(\alpha, \rho, 0,1, G)$ distribution were simulated, $G$ being the log-normal distribution adjusted on Kelly and Rahn's data (Kelly and Rahn, 1932). The GF estimates of $\alpha$ and $\rho$ were calculated with the two distributions $L D(\alpha, \rho, 0,1)$ and $H(\alpha, \rho, 0,1)$. For each couple $(\alpha, \rho)$, the two first boxplots represent the distribution of the $10^{4}$ ratio $\hat{\alpha} / \alpha$ obtained by the $L D$ model (left) and the $H$ model (right); the two last boxplots represent the distribution of the $10^{4}$ ratio $\hat{\rho} / \rho$ obtained by the $L D$ model (left) and the $H$ model (right)

of the estimates obtained with these rectification is smaller than that of the estimates calculated with GF method.

\section{Implementation details}

The available functions are described here; more details are given in the manual. The behavior of inference functions for inputs which are out of practical limitations is described. Some details about the Rcpp implementation are also provided.

\section{User interface}

The flan package can be split into two distinct parts: the distribution of the final number of mutants and statistical inference. The functions dflan, pflan, qflan compute densities, probabilities and quantiles of $L D$ and $H$ distributions. The function $r f l a n$ outputs samples of pairs (mutant counts-final counts) following $L D F N, H F N$, or $M M F N$ where $G$ has a log-normal or gamma distribution and $K$ has a log-normal or Dirac distribution. $K$ is adjusted to the mean and coefficient of variation provided by the user. Those functions have been designed on the principle of the classical distribution functions of R. A graphic function draw. clone is also provided. Using a binary tree, it represents the growth of a clone starting from a single normal cell with mutation occurrences until a finite time. The function mutest im computes estimates of $\alpha$ or $\pi$ and $\rho$, using $L D$ or $H$ models. The three estimation methods are available. Fluctuations of final numbers and cells death are included. The function returns estimate(s) of the parameter(s) of interest and the standard deviations. The function flan. test uses asymptotic normality to perform one or two-sample hypothesis testing. It has been designed on the principle of the classical hypothesis testing functions of R, such as t. test. As mentioned in Section 2.3, there are practical limitations for each estimation method. If the inputs of the mutestim function do not respect those limitations, it will output errors or warning messages:

- If $\delta=0$, the P0 method can not be used if the sample does not contain any null counts. In that 




Figure 6: GF estimates of $\alpha$ ignoring plating efficiency or not. Red horizontal lines mark unit. Blue horizontal lines mark relative bias of 0.9 and 1.1. For each of the 12 sets of parameters $\alpha=(0.5,2,4,8)$ (rows), and $\rho=(0.8,1,1.2)$ (columns), $10^{4}$ samples of size 100 of the $L D(\alpha, \rho, 0, \zeta)$ distribution were simulated, with $\zeta=0.2$ and $\zeta=0.05$. The GF estimates of $\alpha$ and $\rho$ were calculated under $L D$ model. Each boxplot represents the distribution of the $10^{4}$ ratio $\hat{\alpha} / \alpha$ obtained with $\zeta=1$ (left), using the Stewart et al. (1990) correction (center), and with the true value of $\zeta$ (right).

case, the mutestim function will throw an error with a message.

- For now, an inefficient plating (i.e. $\zeta<1$ ) can be taken into account only with the GF method. If another method is specified, the mutestim function will return a warning message and set $\zeta$ at 1 .

- Issues of the Winzorization parameter $w$ (default is 1024) of ML method:

1. If the minimum of the sample is larger than $w$, then the sample of mutant counts will be constant.

2. If $w$ is too large, then the optimization process can be very long.

- The GF method does not have limitations of usage, even for extreme cases where the ML estimators fail, i.e. samples with theoretical large $\alpha$ and small $\rho$. However, estimating $\rho$ requires solving the zero equation discussed in Section 2.3, which is theoretically solvable on $\mathbb{R}^{+}$. In practice the interval of research is bounded. Thus, if the sample does not contain any jackpot, which means that $\rho$ is very large, the zero equation may not have any solution on the interval. In that case, the function will return a warning message, and set the estimate of $\rho$ at 1 , and the estimate of the standard deviation at 0 . In the mutestim function, the domain of research is $[0.01 ; 100]$.

- Moreover, the initialization of the ML method is done with GF method. Then the domain of optimization is $\left[0.1 \times \hat{\theta}_{G F} ; 10 \times \hat{\theta}_{G F}\right]$, where $\hat{\theta}_{G F}$ is the GF estimate(s) of the parameter(s) of interest. Then, if the GF method does not succeed to estimate $\rho$, there is no chance to estimate it with ML. A warning message is returned if the initialization of the estimate of $\rho$ with GF fails.

The function flan. test is a wrapper function of mutestim. It will ouput the same errors or warning messages if its inputs do not respect the practical limitations.

\section{Implementation}

Since most functions involve loops that are more expensive in $\mathrm{R}$ than in $\mathrm{C}$, flan has been implemented with Rcpp modules. This paradigm provides an easy way to expose $\mathrm{C}++$ functions and classes to $\mathrm{R}$. There are four main classes in the $\mathrm{C}++$ implementation: 
- FLAN_Sim: random generation for $M M$ and $M M F N$ distributions. One of its members is a variable of the following type;

- FLAN_SimClone: random generation for clone size distribution according to the lifetimes distribution;

- FLAN_MutationModel: computation of the descriptive functions (probabilities, PGF,...) for $L D$ and $H$ distributions. One of its members is a variable of the following type;

- FLAN_Clone: computation of the descriptive functions for clone size distribution according to the lifetimes distribution.

The Rcpp interface enables also to import into the $\mathrm{C}++$ code any $\mathrm{R}$ function. In particular, it is interesting to import the $\mathrm{R}$ functions which are already implemented in $\mathrm{C}$. Thus no external $\mathrm{C} / \mathrm{C}++$ library is required. The installation remains basic, and the size of the installed package is reduced. For example, the computations of $L D$ distributions involve numeric integrations. The $C$ libraries integration and alglib compute integrals with an accuracy close to machine precision. We could use those libraries but the $\mathrm{R}$ function integrate is actually implemented in $\mathrm{C}$. The $\mathrm{R}$ function can then be directly called into the $\mathrm{C}++$ code. However, such imports increase the computational time and memory consumption. This is thus unsatisfactory. Another solution uses the package RcppGSL (Eddelbuettel, 2013, chap. 11), which creates an interface between Rcpp and the C library gsl. Several integration methods are thus available. Since it only requires that gsl is correctly installed, this solution is a good compromise between easy setup and computational cost.

Computing the probabilities for the $H$ distribution with $\delta>0$ involves squaring high degree polynomials. Such polynomials are easily treated by the package polynom. However its implementation raises memory issues, because of the degree of the polynomials involved. A more efficient way is to use the Fast Fourier Transform. It is provided by the C library fftw3, which can raise some installation issues. The $\mathrm{R}$ function $\mathrm{fft}$ could be directly called into the $\mathrm{C}++$ code. For the same reasons as for the integrate function, it is more adequate to use the package RcppArmadillo (Eddelbuettel (2013, chap.10); Eddelbuettel and Sanderson (2014)). This package links Rcpp to the C++ library armadillo, which is dedicated to linear algebra. In particular, it includes a performant Fast Fourier Transform.

Finally, likelihood optimizations in the ML and P0 methods are done with a bounded BFGS optimizer. The package lbfgsb3 provides the eponymous function which is implemented in Fortran. It is much faster than the basic $\mathrm{R}$ function optim.

\section{Examples of usage}

Some examples on the real data included in flan are provided.

Practical limitations, influence of bias sources and comparison of the estimation methods in terms of computational time are illustrated. Consider first the eleventh sample of mutant counts of the werhoff data (Werngren and Hoffner, 2003):

werhoff\$samples\$W11\$mc



This sample does not contain any jackpot, then the theoretical fitness in a mutation model, should be very large. If the GF method is used, it will output a warning message and set the fitness at $\rho=1$, as customarily done in the litterature (Foster, 2006):






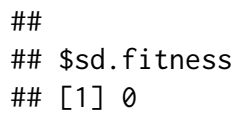

Notice that Table 1 of Werngren and Hoffner (2003) shows mutants counts and mean final numbers of cells for $1 \mathrm{~mL}$ of solution. However, the total volume of the culture is $5 \mathrm{~mL}$. In other words, a plating efficiency of $20 \%$ has been applied. The fact is the fitness parameter can not be estimated, even taking into account the plating efficiency:



Using the P0 method is a way to realize that setting $\rho=1$ by default can be misleading. Since this method does not depend on the lifetime distribution, the estimate of $\alpha$ will not depend on the value of $\rho$.

\# Compute PO estimate of mutations number

The P0 estimate of $\alpha$ is very different from the GF estimate (when $\zeta=1$ ). Consider now the sample of David (1970, Tab. 2), which includes rifampin-resistant bacteria counts.

\section{david\$D11}



Since the 4 value can be seen as a jackpot, the GF method can be used to estimate $\alpha$ and $\rho$. Now let us compute the ML estimates of $\pi$ and $\rho$ taking into account or not of the final counts, under the $L D$ model.

$D<-$ david\$D11

Dmfn $<-\operatorname{mean}(D \$ f n)$

\# Compute ML estimates and confidence intervals of mutation probability and

\# fitness with empirical mean and null coefficient of variation 
$\mathrm{ft}<-$ flan.test $(m c=D \$ m c, m f n=D m f n)$

ft\$estimate

\#\# mutation probability

\#\# 2.067641e-10

fitness

$2.214676 \mathrm{e}+00$

ft\$conf.int



The sample of final counts is denoted by $D_{11}^{(F N)}$. The empirical mean of the final counts is denoted by $\bar{\mu}$, the empirical coefficient of variation by $\bar{C}$. Table 1 displays the ML estimates of $\pi$ and $\rho$, in the same way as for Figure 3. Their $95 \%$ confidence intervals are provided. Comparing the second row to the fourth, one can see that neglecting final number fluctuations induces a bias of order $5 \%$ on $\pi, 10 \%$ on $\rho$. From the third row, it turns out that the correction taking into account $\bar{C}$, has not improved the estimate of $\pi$. Notice also that due to the small size sample, the confidence intervals are quite large. Consider finally the data from Boe et al. (1994, Tab. 4). The author studied mutations of Escherichia coli from sensitivity to nalidixic acid resistance. 23 samples of resistant bacteria counts are provided. As in the original paper, the 23 samples are concatenated as one. The three estimation methods are compared in terms of computational time on the resulting sample of size 1104. The package microbenchmark is used, evaluating $10^{4}$ times each method on the sample. The methods are compared when only $\alpha$ is estimated $(\rho=1)$, and when the couple $(\alpha, \rho)$ is estimated. In both cases the estimates are computed under the model $L D$ with $\delta=0$ and $\zeta=1$.






\begin{tabular}{ccccc}
\hline & Estimates of $\pi$ & $\begin{array}{c}\text { Confidence } \\
\text { intervals }(95 \%) \text { of } \pi\end{array}$ & Estimates of $\rho$ & $\begin{array}{c}\text { Confidence } \\
\text { intervals (95\%) of } \rho\end{array}$ \\
\hline $\begin{array}{c}\text { ML using } \\
\mu=\bar{\mu}, C=0\end{array}$ & $2.07 \times 10^{-10}$ & {$\left[0 ; 4.22 \times 10^{-10}\right]$} & 2.21 & {$[0 ; 8.11]$} \\
\hline $\begin{array}{c}\text { ML using } \\
\mu=\bar{\mu}, C=\bar{C}\end{array}$ & $2.09 \times 10^{-10}$ & {$\left[0 ; 4.51 \times 10^{-10}\right]$} & 2.21 & {$[0 ; 8.11]$} \\
\hline $\begin{array}{c}\text { ML using } \\
D_{11}^{(F N)}\end{array}$ & $1.98 \times 10^{-10}$ & {$\left[0 ; 4.08 \times 10^{-10}\right]$} & 2.05 & {$[0 ; 7.13]$} \\
\hline
\end{tabular}

Table 1: ML estimates of $\pi$ and $\rho$ of data set from (David, 1970, Tab. 2) ignoring fluctuations of final numbers or not. Each row shows the ML estimates of $\pi$ and $\rho$ and their $95 \%$ confidence intervals, deducing from $L D$ model with $C=0$ (first row), $C=\bar{C}$ (second row), and directly with $L D F N$ model (third row).



The results are shown on Figure 7, as boxplots of timing distributions. Times are in milliseconds and plotted on log-scale. As mentioned earlier, the ML method is the slowest. The GF method seems to be quite faster than the P0 method. However, the estimates of $\rho$ of the P0 method is calculated maximizing the likelihood. This step is more costly in term of computational time. If only $\alpha$ has to be estimated, the P0 method is faster than the GF method.

\section{Bibliography}

W. P. Angerer. An explicit representation of the Luria-Delbrück distribution. J. Math. Biol., 42(2): 145-174, 2001a. URL https://doi .org/10.1007/s002850000053. [p335]

W. P. Angerer. A note on the evaluation of fluctuation experiments. Mutation Research, 479:207-224, 2001b. URL https://doi.org/10.1016/S0027-5107(01)00203-2. [p335]

P. Armitage. The statistical theory of bacterial populations subject to mutation. J. R. Statist. Soc. B, 14: 1-40, 1952. URL http://www. jstor.org/stable/2984083. [p334]

K. B. Athreya and P. E. Ney. Branching Processes. Springer-Verlag, Berlin Heidelberg, 1972. URL https://doi.org/10.1007/978-3-642-65371-1. [p334,335, 337] 
Estimates of $\alpha$ setting $\rho=1$



Estimates of the couple $(\alpha, \rho)$



Figure 7: Computational time of the three methods on data from Boe et al. (1994, Tab. 4). Data consist in the 23 samples of boeal concatenated as one. For each method, the estimates of $\alpha$ setting $\rho=1$ (left boxplots) or of $\alpha$ and $\rho$ (right boxplots) have been computed under model $L D$. The timings have been returned with microbenchmark, evaluating $10^{4}$ times each method. Times are in milliseconds and plotted on log-scale.

M. S. Bartlett. An Introduction to Stochastic Processes, with Special Reference to Methods and Applications. Cambridge University Press, $3^{\text {rd }}$ edition, 1978. [p334]

R. Bellman and T. Harris. On age-dependent binary branching processes. Ann. Math., 55(2):280-295, 1952. URL https://www. jstor.org/stable/1969779. [p334, 336]

L. Boe, T. Tolker-Nielsen, K. M. Eegholm, H. Spliid, and A. Vrang. Fluctuation analysis of mutations to nalidixic acid resistance in Escherichia Coli. J. Bacteriol., 176(10):2781-2787, 1994. URL https: //doi.org/10.1128/jb.176.10.2781-2787. [p347,349]

R. Breunig. An almost unbiased estimator of the coefficient of variation. Econ. Lett., 70(1):15-19, 2001. URL https://doi .org/10.1016/S0165-1765(00)00351-7. [p341]

H. L. David. Probability distribution of drug-resistant mutants in unselected populations of Mycobacterium Tuberculosis. Appl. Microbiol., 20(5):810-814, 1970. URL https: //www . ncbi .nlm. nih. gov/pmc/ articles/PMC377053/. [p346, 348]

A. Dewanji, E. G. Luebeck, and S. H. Moolgavkar. A generalized Luria-Delbrück model. Math. Biosci., 197(2):140-152, 2005. URL https://doi.org/10.1016/j.mbs.2005.07.003. [p335]

D. Eddelbuettel. Seamless R and C++ Integration with Rcpp. Springer-Verlag, New York, 2013. URL https://doi.org/10.1007/978-1-4614-6868-4. [p335,345]

D. Eddelbuettel and C. Sanderson. Rcpparmadillo: Accelerating R with high-performance C++ linear algebra. Comput. Stat. Data An., 70:1054-1063, 2014. URL https://doi .org/10. 1016/j . csda. 2013. 02.005. [p345]

P. Embrechts and J. Hawkes. A limit theorem for the tails of discrete infinitely divisible laws with applications to fluctuation theory. J. Austral. Math. Soc. Series A, 32:412-422, 1982. URL https: //doi.org/10.1017/S1446788700024976. [p337]

P. L. Foster. Methods for determining spontaneous mutation rates. Method. Enzymol., 409:195-213, 2006. URL https://doi.org/10.1016/s0076-6879(05)09012-9. [p345]

A. Gillet-Markowska, G. Louvel, and G. Fisher. Bz-rates: a web-tool to estimate mutation rates from fluctuation analysis. G3, 5(11):2323-2327, 2015. URL https://doi .org/10.1534/g3.115.019836. [p335] 
B. M. Hall, C. X. Ma, P. Liang, and K. K. Singh. Fluctuation AnaLysis CalculatOR: a web tool for the determination of mutation rate using Luria-Delbrück fluctuation analysis. Bioinformatics, 25(12): 1564-1565, 2009. URL https://doi.org/10.1093/bioinformatics/btp253. [p335]

A. Hamon and B. Ycart. Statistics for the Luria-Delbrück distribution. Elect. J. Statist., 6:1251-1272, 2012. URL https://doi .org/10.1214/12-EJS711. [p334, 335, 336, 337, 338]

C. D. Kelly and O. Rahn. The growth rate of individual bacterial cells. J. Bacteriol., 23(2):147-153, 1932. URL http://europepmc.org/articles/PMC533308. [p343]

N. L. Komarova, L. Wu, and P. Baldi. The fixed-size Luria-Delbrück model with a nonzero death rate. Math. Biosci., 210(1):253-290, 2007. URL https://doi .org/10.1016/j . mbs. 2007.04.007. [p335]

D. E. Lea and C. A. Coulson. The distribution of the number of mutants in bacterial populations. Journal of Genetics, 49(3):264-285, 1949. URL https: //doi .org/10.1007/BF02986080. [p334]

S. E. Luria and M. Delbrück. Mutations of bacteria from virus sensitivity to virus resistance. Genetics, 28(6):491-511, 1943. URL https://www. genetics.org/content/28/6/491. [p334, 337]

W. T. Ma, G. v. H. Sandri, and S. Sarkar. Analysis of the Luria-Delbrück distribution using discrete convolution powers. J. Appl. Probab., 29(2):255-267, 1992. URL https://doi .org/10.1017/ S0021900200043023. [p334]

B. Rémillard and R. Theodorescu. Inference based on the empirical probability generating function for mixtures of Poisson distributions. Statist. Decisions, 18:349-366, 2000. URL https://doi . org/10. 1524/strm. 2000.18.4.349. [p334, 338]

S. Sarkar. Haldane's solution of the Luria-Delbrück distribution. Genetics, 127:257-261, 1991. [p334]

F. M. Stewart. Fluctuation analysis: The effect of plating efficiency. Genetica, 84(1):51-55, 1991. URL https://doi.org/10.1007/BF00123984. [p335]

F. M. Stewart, D. M. Gordon, and B. R. Levin. Fluctuation analysis: The probability distribution of the number of mutants under different conditions. Genetics, 124(1):175-185, 1990. URL https: //www. genetics.org/content/124/1/175. [p335, 337, 342, 344]

J. Werngren and S. E. Hoffner. Drug susceptible Mycobacterium Tuberculosis Beijing genotype does not develop motation-conferred resistance to Rifampin at an elevated rate. J. Clin. Microbiol., 41(4): 1520-1524, 2003. URL https://doi.org/10.1128/jcm.41.4.1520-1524.2003. [p345, 346]

R. Wilcox. Introduction to Robust Estimation and Hypothesis Testing. Elsevier, Amsterdam, $3^{\text {rd }}$ edition, 2012. URL https://doi .org/10.1016/c2010-0-67044-1. [p334, 337]

B. Ycart. Fluctuation analysis: Can estimates be trusted? PLoS One, 8(12):1-12, 2013. URL https: //doi.org/10.1371/journal. pone.0080958. [p334]

B. Ycart. Fluctuation analysis with cell deaths. J. Appl. Probab. Statist, 9(1):13-29, 2014. URL https: //arxiv.org/abs/1207.4375. [p335, 337]

B. Ycart and N. Veziris. Unbiased estimates of mutation rates under fluctuating final counts. PLoS One, 9(7):1-10, 2014. URL https://doi.org/10.1371/journal. pone.0101434. [p335, 337, 340]

Q. Zheng. Statistical and algorithmic methods for fluctuation analysis with SALVADOR as an implementation. Math. Biosci., 176(2):237-252, 2002. URL https://doi.org/10.1016/s0025$5564(02) 00087-1$. [p335]

Q. Zheng. New algorithms for Luria-Delbrück fluctuation analysis. Math. Biosci., 196(2):198-214, 2005. URL https://doi.org/10.1016/j.mbs.2005.03.011. [p334,337]

Adrien Mazoyer

Statistique pour les sciences du Vivant et de l'Homme, Laboratoire Jean Kuntzmann

Université Grenoble Alpes

Bâtiment IMAG, 700 Avenue Centrale

38400 SAINT MARTIN D'HÈRES

FRANCE

adrien.mazoyer@univ-grenoble-alpes.fr

Rémy Drouilhet

Fiabilité et Géométrique Aléatoire, Laboratoire Jean Kuntzmann 
Université Grenoble Alpes

Bâtiment IMAG, 700 Avenue Centrale

38400 SAINT MARTIN D'HÈRES

FRANCE

remy.drouilhet@univ-grenoble-alpes.fr

Stéphane Despréaux

Laboratoire Jean Kuntzmann

Université Grenoble Alpes

Bâtiment IMAG, 700 Avenue Centrale

38400 SAINT MARTIN D'HËRES

FRANCE

stephane. despreaux@univ-grenoble-alpes.fr

Bernard Ycart

Statistique pour les sciences du Vivant et de l'Homme, Laboratoire Jean Kuntzmann

Université Grenoble Alpes

700 Avenue Centrale, 38400 SAINT MARTIN D'HÈRES

FRANCE

bernard.ycart@univ-grenoble-alpes.fr 